\title{
Sever's Disease of the Pediatric Population: Clinical, Pathologic, and Therapeutic Considerations
}

\author{
Mohamad Y. Fares, MD, MRes; Hamza A. Salhab, MD, MRes; Hussein H. Khachfe, MD; \\ Jawad Fares, MD, MSc; Rachid Haidar, MD; and Umayya Musharrafieh, MD, MSc
}

\begin{abstract}
Sever's disease is an underreported prevalent pediatric condition that causes heel pain in children worldwide. It is often described as an overuse injury that can present with either unilateral or bilateral heel pain. Even though the exact mechanism of injury is unknown, it is often thought it involves repetitive stress and pressure on the calcaneal growth plate. Diagnosing Sever's disease mainly relies on a thorough clinical investigation and physical examination, with a positive squeeze test usually sufficient to establish diagnosis. Nevertheless, radiographic imaging can help exclude other differential diagnoses. Therapeutic options of Sever's disease are mostly conservative, and these include rest, physical therapy, kinesiotherapy, and orthoses. Educating parents and coaches on the symptomatology and presentation of Sever's disease is pivotal for the establishment of efficient preventive interventions and earlier diagnoses. This study presents a case of a pediatric patient with Sever's disease and offers medical insight into the diagnostic, clinical, pathologic, and therapeutic characteristics of this condition, in light of the current existing literature.
\end{abstract}

Keywords: Sever's disease; Calcaneal apophysitis; Pediatrics: Overuse injury

$\mathrm{O}$ $\mathrm{f}$ all the musculoskeletal complaints in the pediatric population, posterior heel pain is considered the most common, especially in active children between ages 8 and 15 years. ${ }^{1,2}$ One prominent culprit of pediatric heel pain is Sever's disease, or calcaneal apophysitis, a condition first described by James Warren Sever ${ }^{3}$ in 1912, with a reported incidence of 3.7 per 1000 patients. ${ }^{4}$ This condition is often attributed to calcaneal inflammation in a physically active growing child. ${ }^{5}$ It is described as an overuse injury with an insidious onset of pain, and is often not related to a traumatic event. ${ }^{5}$ Presentation and etiologies can differ according to individual case, but associations with physical activity and weight bearing have been established..$^{5}$

A proper clinical investigation, which includes comprehensive history taking and a thorough physical exam, is needed to diagnose Sever's disease. ${ }^{6}$ In most cases, a positive heel squeeze test is usually sufficient and indicative of the underlying pathology; however, radiographic imaging can help by excluding other differential diagnoses. ${ }^{5,7,8}$ Treatment options are almost always conservative and vary between rest, physical therapy, kinesiotherapy, orthotics and others. ${ }^{5}$ Despite its prevalence in the pediatric community, not many studies on the characteristics of Sever's disease exist in the medical literature. Understanding the anatomical, diagnostic, and therapeutic characteristics of this disease is key for devising better treatment plans and establishing efficient prevention strategies. Accordingly, the aim of this study is to present a case of Sever's disease in a boy, aged 11 years, and discuss the clinical features of Sever's disease from diagnostic, clinical, pathologic, and therapeutic perspectives, based on a thorough literature review.
Corresponding Author: Mohamad Y. Fares, MD, MRes, College of Medical, Veterinary, and Life Sciences, University of Glasgow, Glasgow, Scotland, UK, Email: 2423564F@student.gla.ac.uk
Received: November I, 2020

Revised: April 26, 2021

Accepted: May II, 202 I

doi: $|0.3| 2 \mid / \mathrm{cmr} .2021 .1639$ 


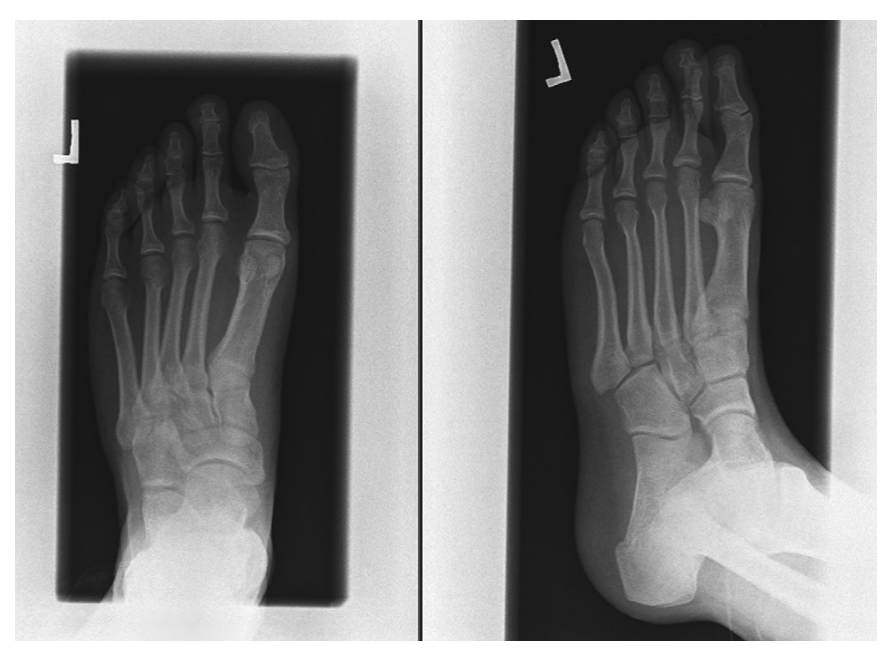

Figure 1. Radiographic imaging (X-ray) of the patient revealing no abnormalities or fractures.

\section{Case Presentation}

A boy, aged 11 years, presented to the clinic with a complaint of unilateral left foot pain of one month duration. The patient was previously healthy, of average weight and height, and does not take any medications. He could not recall any traumatic or inciting event that precipitated his injury. The pain was at the level of the posterior heel and was exacerbated following heavy exercise. The patient was described as very active and vigilant prior to the complaint; however, his parent reported that the pain progressively increased in intensity to the extent that forced him to stop participating in sports. Differential diagnoses at that point included Sever's disease, retrocalcaneal bursitis, Achilles tendonitis, osteomyelitis, and plantar fasciitis.

On physical examination, the patient had a noticeable limp. There was no significant erythema or swelling involving the

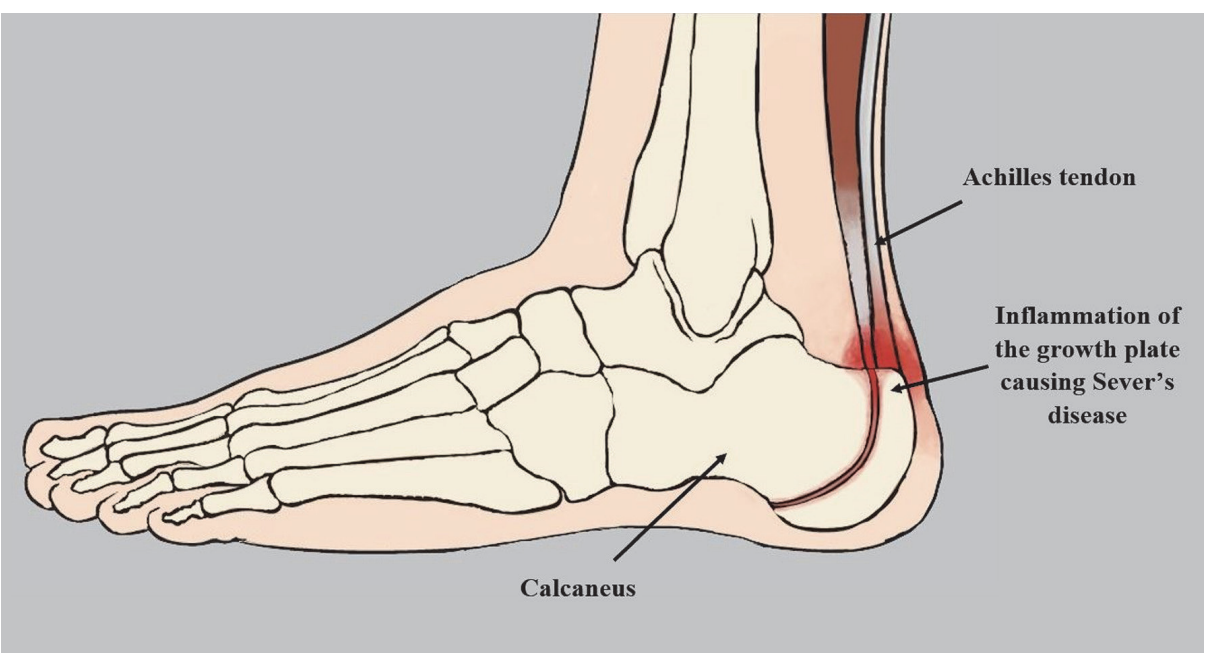

affected area, and sensation and motor power of the foot were intact. Palpation revealed severe tenderness of the posterolateral aspect of the left heel. A positive heel squeeze test entertained the diagnosis of Sever's disease. Radiographic imaging was performed and revealed no abnormalities or fractures, further confirming the suspected diagnosis (Figure 1). The patient was treated conservatively with rest, ice application, and stretching exercises. One month later, the patient reported improvement of symptoms and normal return to daily activities.

\section{Clinical Anatomy and Biomechanics}

The calcaneus is the largest bone in the foot, positioned at the most plantar posterior aspect. ${ }^{2}$ It is associated distally with the cuboid and superiorly with the talus to constitute part of the midtarsal joint. ${ }^{2,8}$ The insertion of three muscles takes place at the calcaneus: the gastrocnemius, the soleus, and the plantaris. The insertion of the Achilles tendon, along with other muscles and ligaments necessary for walking, takes place in the lower posterior side of the calcaneus (Figure 2). ${ }^{5}$ Adjacent to the insertion of the Achilles tendon exists the apophysis, a bony growth center subject to prominent axial load. ${ }^{2}$

In the pediatric patient, the apophysis is considered the weakest point in the muscle-tendon-bone attachment, as opposed to the tendon in adults. ${ }^{10}$ Its growth plate remains open until at least age 14 years. ${ }^{5}$ The early adolescent growth spurt causes bone growth to exceed that of the muscle-tendon unit. ${ }^{11}$ As a result, the muscle-tendon unit loses the ability to stretch sufficiently to maintain its previous flexibility. ${ }^{5}$ This, in turn, causes increased tension across the incompletely-ossified apophysis. This stress to the calcaneal area can irritate the growth plate, and consequently, cause inflammation. ${ }^{5}$

Many debates exist regarding the exact mechanism that governs Sever's disease. One main theory attributes the disease to the mechanical overuse resulting from ongoing shear stress and repetitive impact pressure to the open calcaneal growth plate. ${ }^{5}$ Previous research addressed the role of plantar loading in this condition and reported higher peak plantar pressures beneath the heel in pediatric patients with Sever's disease. ${ }^{12}$ A study by Becerro de Bengoa Vallejo et $\mathrm{al}^{12}$ reported peak plantar pressures of $339+/-27 \mathrm{kPa}$ in Sever's disease patients during standing, compared to $83+/-2 \mathrm{kPa}$ in healthy controls. During walking, reported peak plantar pressures reached $880+/-78$ $\mathrm{kPa}$ in Sever's disease patients, and $88+/-11 \mathrm{kPa}$ in healthy controls. ${ }^{12} \mathrm{As}$

Figure 2. Anatomy of Sever's disease. 
such, one could understand why sports that involve walking, running, and jumping cause the most pain, as plantar loads tend to increase during these activities. ${ }^{13}$

Running is, in fact, mainly associated with Sever's disease, as high exposure in children results in muscular imbalances. ${ }^{14,15}$ These imbalances manifest through strong plantar flexors and knee extensor muscles and weak knee flexors and dorsiflexors. ${ }^{15}$ These imbalances cause an increased strain on the bone during physical activity, and predisposes to Sever's disease. ${ }^{13,14}$ In addition, a study showed that children with Sever's disease have increased normalized cadence while running, possibly in an attempt to decrease the peak plantar pressures and subsequent pain. ${ }^{16}$ This further suggests the importance of assessing running in children with symptomatic calcaneal apophysitis, as opposed to normal walking gait. Additional research is needed to accurately describe and uncover the mechanisms that govern this condition.

\section{Presentation and Risk Factors}

Sever's disease often presents in children around age 8-15 years, accompanied by heel pain and tenderness that is exacerbated by physical activity. ${ }^{5}$ Patients often cannot recall a specific injury that caused their pain. ${ }^{5}$ The pain is mostly non-radiating, often located over the posterior aspect of the heel, adjacent to the insertion of the Achilles tendon. ${ }^{2}$ Erythema and swelling are usually absent, and patients often present with limited ankle dorsiflexion. ${ }^{5}$ The condition can present unilaterally or bilaterally, and patients can be seen with an adjusted gait, walking on their toes or with a limp, to avoid bearing weight on the affected foot. ${ }^{17}$ Differential diagnoses include osteomyelitis, a condition that can cause deleterious outcomes like growth arrest. ${ }^{5,18}$

Risk factors to Sever's disease involve variables that cause an increased strain and load to the posterior heel, ${ }^{14}$ which includes running and jumping sports. One study from 2014 showed around $87 \%$ of young athletes that suffer from heel pain are enrolled in running or jumping sports. ${ }^{19}$ Other reported risk factors include improper footwear and equipment, a greater body mass index, greater waist circumference, higher weight, and increased height. ${ }^{20-22}$ Studies also show a greater prevalence among males, with some reporting a 2-3 folds discrepancy. ${ }^{14,21}$ This is often attributed to the delayed skeletal maturity in males when compared to females. ${ }^{23}$

\section{Diagnosis}

Diagnosis of Sever's disease mainly relies on a thorough clinical investigation, and a focused physical examination. ${ }^{14}$ Common symptoms such as posterior heel pain and impaired gait during walking or running should be evaluated promptly. ${ }^{14}$ In addition, a history that involves sports participation, as well as an exacerbation with physical activity should raise suspicion with regards to this pathology. ${ }^{5}$ Ankle examination should include an assessment of passive and active ankle range of motion and an inspection for physical deformities, erythema, swelling, or bruising. ${ }^{24}$ Meticulous palpation of the ankle is pivotal in identifying the designated pathology and is necessary for localizing areas of tenderness. ${ }^{24}$ In addition, the squeeze test is considered one of the most important components of the physical examination in the setting of Sever's disease. ${ }^{25}$ This is the most common clinical test for diagnosing Sever's disease and involves the compression of the medial and lateral aspects of the heel (Figure 3). ${ }^{25} \mathrm{~A}$ positive result solicits pain.

Much debate exists on whether imaging is necessary for diagnosing Sever's disease. ${ }^{7}$ Nevertheless, it is often accepted that imaging can assist in ruling out other differential diagnoses. Radiographic imaging or ultrasound may show fragmentation of the secondary calcaneal nucleus. ${ }^{26}$ Magnetic resonance imaging can visualize the apophysis and the pull of the Achilles tendon. ${ }^{26}$ It can also show edema at the level of the calcaneus or heel pad and exclude other conditions like stress fractures, small bone fusion, and osteomyelitis. ${ }^{26}$

\section{Treatment}

Treatment of Sever's disease aims at supporting tissue healing, strengthening the area associated with the pathology, and allowing a return to sports and physical activity with full recovery. ${ }^{2}$ The ambiguity behind the pathophysiological mechanisms of Sever's disease entails a variation in treatment modalities. The available treatment modalities currently used are mainly conservative and include: physical therapy and

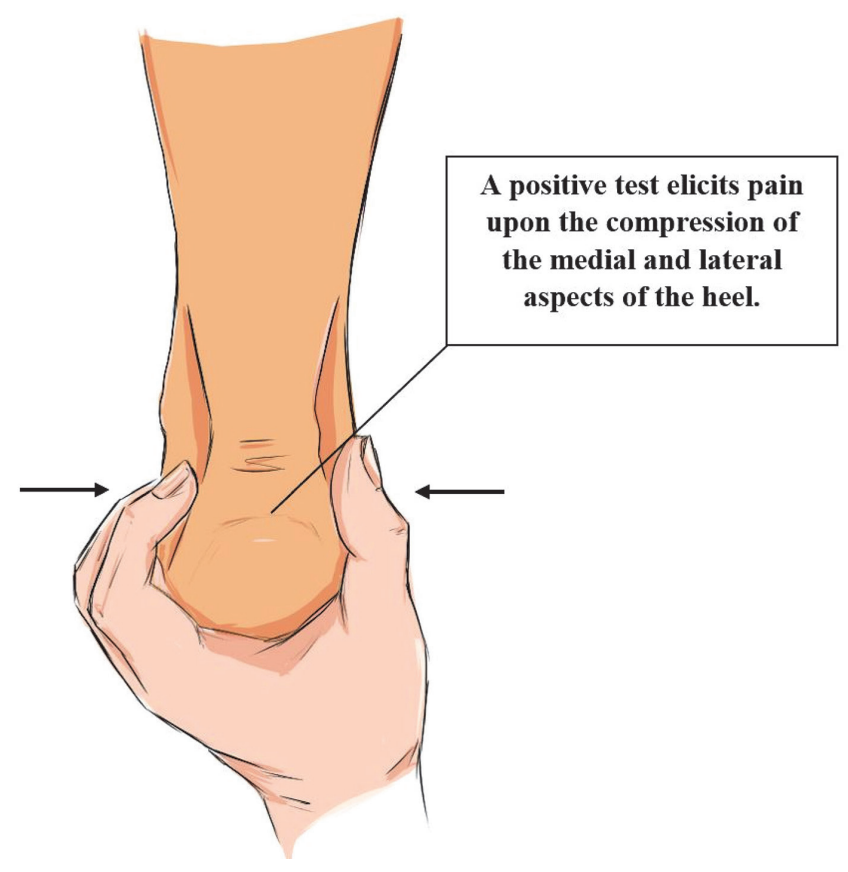

Figure 3. The calcaneal squeeze test. 
rest, kinesiotherapy and taping, and orthotic devices. ${ }^{5}$ Treatment regimens should tackle an initial phase of recovery and rest followed by stretching and strengthening. ${ }^{2}$ Kinesiotherapy and foot orthoses can further help in reducing pain, restoring function, and improving biomechanical outcomes.

\section{Physical Therapy and Rest}

The use of ice, activity restriction, and stretching and strengthening exercises have been incorporated in the majority of studies exploring Sever's disease. ${ }^{5}$ Decreasing the amplitude of the inflammatory process occurring at the calcaneal apophysis can prominently decrease pain and improve outcomes. ${ }^{27}$ Oral and topical non-steroidal antiinflammatory drugs (NSAIDs) have been used for the reduction of pain in some reports. ${ }^{28}$ Although, while the use of the topical NSAID ketoprofen is contraindicated in children younger than age 12 years, one case report utilized this treatment under close medical monitoring for adverse effects and reported positive outcomes. ${ }^{28}$

Physical therapy mainly consists of stretching and strengthening protocols. Therapy protocols often work on stretching the gastrocnemius muscle, since it provides direct traction on the Achilles tendon and causes subsequent tension on the heel. ${ }^{29}$ As mentioned earlier, during growth spurts, the bony structures in the heel grow faster than the muscles and tendons, and this causes increased pull from the calf muscle onto the Achilles tendon. ${ }^{9}$ Stretching exercises can help alleviate the resultant pain. In addition, strengthening exercises are necessary later on to preserve function and improve outcomes, as excessive rest and immobilization can result in weak lower limb muscles. Eccentric strengthening via heel raises is usually used to regain range of motion. ${ }^{30}$ Exercises are often applied without weight-bearing loads, which can then be increased as pain subsides. Nevertheless, caution should be taken when performing the strengthening exercises, as these often promote increased dorsiflexion stretching and plantar flexion contraction. If applied at the wrong phase of recovery, these exercises can increase the stress on the Achilles tendon and exacerbate the pain.

\section{Kinesiotherapy and Taping}

Few studies used taping in the management of Sever's disease patients. The principle mechanism behind kinesiotherapy and taping is to regulate blood flow, eliminate inflammatory cytokines in the region, and reduce muscular tension and stress, causing pain relief. ${ }^{31}$ This modality has been found effective in providing acute pain relief in the immediate setting. One case series by Hunt et $\mathrm{al}^{32}$ assessed heel pain levels in pediatric patients with Sever's disease before and after tape application. The study showed significant pain reduction immediately after applying the tape. ${ }^{32}$ However, another study conducted by Kuyucu et $\mathrm{al}^{33}$ showed no significant reduction in pain levels after being treated with kinesiotherapy. Kuyucu et $\mathrm{al}^{33}$ divided pediatric patients with Sever's disease into two groups: one that was administered actual kinseiotherapy, and another that was administered sham therapy. Even though the kinesiotherapy group showed improved functional outcomes, there was no significant difference in pain levels between the taping group and the sham group. ${ }^{32}$

\section{Orthoses}

Orthoses are often used in the treatment of Sever's disease in the pediatric population. These provide a cushion to the heel that helps absorb the impact of the ground during jumping or running. ${ }^{34}$ They also lift the heel around half an inch, subsequently placing the foot at plantar flexion. ${ }^{28}$ This relaxes the Achilles tendon and lowers the pressure on the calcaneus, leading to a decrease in pain. ${ }^{2}$ Orthoses can prove beneficial in patients whose symptoms are bothersome, but not enough to warrant stopping their sports activities. ${ }^{10,35}$

Orthoses have been found to be just as effective as other therapeutic protocols in alleviating heel pain. One study by Perhamre et $\mathrm{al}^{10}$ showed that after treating patients with heel cups for a period of 4 weeks, pain scores reached 0 on a $0-10$ Likert scale $(0=$ no pain; $10=$ worst pain $)$. Another study compared the use of orthoses to that of physical therapy and of rest in the management of Sever's disease. ${ }^{36}$ All three modalities caused significant reduction in pain levels with no significant differences between them. Orthoses are often integrated with physical therapy and rest to provide more favorable outcomes.

\section{Prevention}

Reducing the prevalence of this condition is necessary from both economic and medical perspectives, especially given that around $50 \%$ of overuse injuries in youth are reported to be preventable. ${ }^{37}$ Preventive measures should aim at identifying risk factors and adjusting them as per the individual patient. ${ }^{37}$ This can be done via educational, environmental, and physical interventions. ${ }^{38}$ Coaches and parents should be educated on the presentation and causes of Sever's disease in children to help diagnose the condition earlier and avoid long recovery periods and layoffs. ${ }^{39}$ In addition, coaches and parents should ensure the footwear and equipment used by the children are properly fit and provide firm support to avoid biomechanical disruption of the heel and consequent pain. ${ }^{19}$ Furthermore, pediatric training regimens should take into account the risk of exhaustion and should integrate rest periods to allow for recovery. ${ }^{40}$ These regimens should also minimize running-based training, and instead prioritize motor skill development and general fitness. ${ }^{19}$ This allows the children to garner the benefits of exercise with a reduced risk of overuse injuries. Finally, preventive exercise that focuses on improving muscular strength and endurance, coordination, balance, and flexibility can help in reducing the risk of Sever's disease. ${ }^{14}$ These often 
include focused hamstring-to-heel regimens that should be performed after a proper warm-up to avoid muscle pulls. ${ }^{17}$

\section{Conclusion}

Sever's disease is an overuse condition that affects many children worldwide. It often presents with heel pain that can be unilateral or bilateral in nature. Even though the exact mechanism of injury is unknown, it is often speculated to be caused by repetitive stress and pressure on the calcaneal growth plate. Clinical investigation and physical examination are often sufficient to diagnose Sever's disease, and radiographic imaging can be utilized to exclude other differential diagnoses. Treatment options are mostly conservative and include rest, physical therapy, kinesiotherapy, and orthoses. It is important to educate parents and coaches on the symptomatology and etiology of Sever's disease to allow for efficient preventive interventions and earlier diagnoses.

\section{References}

1. Volpe R. Keys to diagnosing and treating calcaneal apophysitis. Podiatry Today. 2009;22(11):60-66.

2. Howard R. Diagnosing and treating Sever's disease in children. Emerg Nurse. 2014;22(5):28-30.

3. Sever JW. Apophysis of os calcis. NY State J Med. 1912;95:1025.

4. Wiegerinck JI, Yntema C, Brouwer HJ, Struijs PA. Incidence of calcaneal apophysitis in the general population. Eur J Pediatr. 2014;173(5):677-679.

5. Ramponi DR, Baker C. Sever's Disease (Calcaneal Apophysitis). Adv Emerg Nurs J. 2019;41(1):10-14.

6. Madden CC, Mellion MB. Sever's disease and other causes of heel pain in adolescents. Am Fam Physician. 1996;54(6):1995-2000.

7. Rachel JN, Williams JB, Sawyer JR, Warner WC, Kelly $\mathrm{DM}$. Is radiographic evaluation necessary in children with a clinical diagnosis of calcaneal apophysitis (sever disease)?. Pediatr Orthop. 2011;31(5):548-550.

8. Kose O, Celiktas M, Yigit S, Kisin B. Can we make a diagnosis with radiographic examination alone in calcaneal apophysitis (Sever's disease)? J Pediatr Orthop B. 2010;19(5):396-398.

9. Scharfbillig RW, Jones S, Scutter SD. Sever's disease: what does the literature really tell us?. Am Podiatr Med Assoc. 2008;98(3):212-223.

10. Perhamre S, Janson S, Norlin R, Klässbo M. Sever's injury: treatment with insoles provides effective pain relief. Scand J Med Sci Sports. 2011;21(6):819-823.

11. Smith JM, Varacallo M. Sever's Disease. [Updated 2021 Jun 7]. In: StatPearls [Internet]. Treasure Island (FL): StatPearls Publishing; 2021 Jan. Available at: https:// www.ncbi.nlm.nih.gov/books/NBK441928/. Last accessed: August 23, 2021.
12. Becerro de Bengoa Vallejo R, Losa Iglesias ME, Rodríguez Sanz D, Prados Frutos JC, Salvadores Fuentes P, Chicharro JL. Plantar pressures in children with and without sever's disease. J Am Podiatr Med Assoc. 2011;101(1):17-24.

13. Orendurff MS, Rohr ES, Segal AD, Medley JW, Green III JR, Kadel NJ. Regional foot pressure during running, cutting, jumping, and landing. Am J Sports Med. 2008;36(3):566-571.

14. Naaktgeboren K, Dorgo S, Boyle JB. Growth Plate Injuries in Children in Sports: A Review of Sever's Disease. Strength Condit J. 2017;39(2):59-68.

15. Kennedy JG, Knowles B, Dolan M, Bohne W. Foot and ankle injuries in the adolescent runner. Curr Opin Pediatr. 2005;17(1):34-42.

16. McSweeney S, Reed LF, Wearing SC. Vertical ground reaction forces during gait in children with and without calcaneal apophysitis. Gait Posture. 2019;71:126-130.

17. Hendrix CL. Calcaneal apophysitis (Sever disease). Clin Podiatr Med Surg. 2005;22(1):55-62, vi.

18. Mustapi? M, Bori? I, Lepur D, Zadravec D, Viskovi? K. Sever's disease complicated with osteomyelitis. Acta Clin Croat. 2014;53(2):252-255.

19. Becerro-de-Bengoa-Vallejo R, Losa-Iglesias ME, Rodriguez-Sanz D. Static and dynamic plantar pressures in children with and without sever disease: a casecontrol study. Phys Ther. 2014;94(6):818-826.

20. Micheli LJ, Ireland ML. Prevention and management of calcaneal apophysitis in children: an overuse syndrome. J Pediatr Orthop. 1987;7(1):34-38.

21. James AM, Williams CM, Luscombe M, Hunter R, Haines TP. Factors associated with pain severity in children with calcaneal apophysitis (sever disease). J Pediatr. 2015;167(2):455-459.

22. DiFiori J. Evaluation of overuse injuries in children and adolescents. Curr Sports Med Rep. 2010;9(6):372-378.

23. Frush TJ, Lindenfeld TN. Peri-epiphyseal and Overuse Injuries in Adolescent Athletes. Sports Health. 2009;1(3):201-211.

24. Pommering TL, Kluchurosky L, Hall SL. Ankle and foot injuries in pediatric and adult athletes. Prim Care. 2005;32(1):133-161.

25. Toomey P. Plantar heel pain. Foot Ankle Clin. 2009;14(2):229-245.

26. Chang G, Paz D, Dwek J, Chung C. Lower extremity overuse injuries in pediatric athletes: Clinical presentation, imaging, findings and treatment. Clin Imaging. 2013;37(5):836-846.

27. James AM, Williams CM, Haines TP. Effectiveness of interventions in reducing pain and maintaining physical activity in children and adolescents with calcaneal apophysitis (Sever's disease): a systematic review. J Foot Ankle Res. 2013;6(1):16. 
28. White RL. Ketoprofen gel as an adjunct to physical therapist management of a child with Sever disease. Phys Ther. 2006;86(3):424-433.

29. Elengard T, Karlsson J, Silbernagel KG. Aspects of treatment for posterior heel pain in young athletes. Open Access J Sports Med. 2010;1:223-232. Published 2010 Dec 6.

30. Carcia CR, Martin RL, Houck J, Wukich DK. Achilles pain, stiffness, and muscle power deficits: Achilles tendonitis. J Orthop Sports Phys Ther. 2010;40(9):A1A26.

31. Kase K, Wallis J, Kase T. Clinical therapeutic applications of the Kinesio taping method. Albuquerque, NM:Kinesio; 2003.

32. Hunt GC, Stowell T, Alnwick GM, Evans S. Arch taping as a symptomatic treatment in patients with Sever's disease: a multiple case series. Foot. 2007;17(4):178-183.

33. Kuyucu E, Gülenç B, Biçer H, Erdil M. Assessment of the kinesiotherapy's efficacy in male athletes with calcaneal apophysitis. J Orthop Surg Res. 2017;12(1):146.

34. James AM, Williams CM, Haines TP. Effectiveness of footwear and foot orthoses for calcaneal apophysitis: a 12-month factorial randomised trial. Br J Sports Med. 2016;50(20):1268-1275.

35. Weiner DS, Morscher M, Dicintio MS. Calcaneal apophysitis: simple diagnosis, simpler treatment. J Fam Pract. 2007;56(5):352-355.

36. Wiegerinck JI, Zwiers R, Sierevelt IN, van Weert HC, van Dijk CN, Struijs PA. Treatment of calcaneal apophysitis: wait and see versus orthotic device versus physical therapy: a pragmatic therapeutic randomized clinical trial. J Pediatr Orthop. 2016;36(2):152-157.

37. Parkkari J, Kujala UM, Kannus P. Is it possible to prevent sports injuries? Sports Med. 2001;31(14):985995.

38. Adirim T and Cheng T. Overview of injuries in the young athlete. Sports Med. 2003;33(1):75-81.

39. Williams JM, Wright $P$, Currie CE, Beattie TF. Sports related injuries in Scottish adolescents aged 11-15. Br J Sports Med. 1998;32(4):291-296.

40. Johnson JH. Overuse injuries in young athletes: Cause and prevention. Strength and Conditioning Journal. 2008;30(2):27-31. Available at: https://excelsiorgroup. co.uk/wp-content/uploads/2015/03/overuse-youth-1.pdf. Last accessed: August 23, 2021.

\section{Author Affiliations}

Mohamad Y. Fares, MD, MSc *; Hamza A. Salhab, MD, MSc, ; Hussein H. Khachfe, MD ${ }^{*}$; Jawad Fares, MD,

MSc ${ }^{\S}$; Rachid Haidar, MD"; and Umayya Musharrafieh, $M D, M S c^{t}$

*Neuroscience Research Center, Faculty of Medicine, Lebanese University, Beirut, Lebanon College of Medical, Veterinary, and Life Sciences, University of Glasgow, Glasgow, Scotland, UK

${ }^{*}$ Faculty of Medicine, American University of Beirut, Beirut, Lebanon

${ }^{\S}$ Department of Neurological Surgery, Feinberg School of Medicine, Northwestern University, Chicago, IL 60611, USA "Department of Orthopedics, American University of Beirut Medical Center, Beirut, Lebanon. 